\title{
Predictors of hospital re-admissions among Hispanics with hepatitis C-related cirrhosis
}

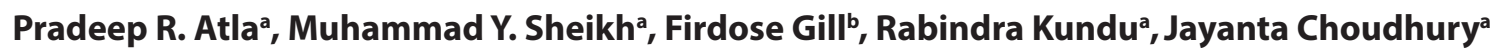 \\ University of California San Francisco, Fresno MEP; Kaiser Permanente Fresno Medical Center, Fresno, California, USA
}

\section{Abstract}

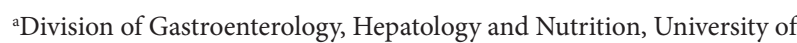
California San Francisco, Fresno MEP (Pradeep R. Atla, Muhammad Y. Sheikh, Rabindra Kundu, Jayanta Choudhury); ${ }^{b}$ Department of Medicine, Kaiser Permanente Fresno Medical Center (Firdose Gill), Fresno, California, USA

Conflict of Interest: None

Correspondence to: Pradeep R. Atla, MD, MPH, Division of Gastroenterology, Hepatology and Nutrition, University of California San Francisco, Fresno, 2823 Fresno str., $1^{\text {st }}$ floor, Endoscopy suite, Fresno, California 93721, USA, Tel.: +1 559459 3882,

Fax: +1 559459 3887, e-mail: patla@fresno.ucsf.edu

Received 17 May 2016; accepted 23 June 2016; published online 21 July 2016

DOI: http://dx.doi.org/10.20524/aog.2016.0072

\section{Introduction}

The Hispanic population is the largest and fastest growing minority group in the United States (US), comprising about $15 \%$ of the US population; by the year 2050 Hispanics are projected to make up about $30 \%$ of the population [1]. Data from the United States National Center for Health Statistics (2007) identified chronic liver disease as the sixth most common cause of death in the Hispanic population, in contrast to non-Hispanic Whites and African Americans, for whom liver disease is not included in the top 10 most frequent causes of death [2]. While mortality rates from chronic liver disease in the United States have progressively declined during the past decade, this trend was not seen in the Hispanic population [3]. 
Hepatitis C virus (HCV) is one of the leading causes of chronic liver disease and rates of HCV mortality are increasing, despite the overall decline in mortality from cirrhosis [3-5]. It has been projected that an aging population of chronically infected patients will lead to a doubling of the proportion of HCV patients with cirrhosis from $16 \%$ to $32 \%$ by 2020 [6]. Data have shown that Hispanics have a higher prevalence [7], mortality rate $[3,5]$, and faster rate of progression of $\mathrm{HCV}$ compared to other ethnic groups [8]. The Hepatitis C Antiviral Long-term Treatment Against Cirrhosis (HALT-C) trail has shown that Hispanics have a higher rate of de novo varices [9], and several studies have demonstrated that the presence of esophageal varices in patients with HCV cirrhosis is a predictor of mortality and decompensated liver disease $[10,11]$.

Cirrhosis, like other chronic disease states, is responsible for significant morbidity and healthcare costs [12,13]. A high frequency of admissions has been recognized as an important factor affecting the rise in healthcare costs in the United States. The estimated cost of unplanned readmissions was $\$ 17.4$ billion in 2004, which comprised 20\% of Medicare's hospital payments. Therefore, readmission rates have been proposed as a national quality indicator and the search for modifiable risk factors for readmissions is considered critical to limiting healthcare spending [14]. Recent studies have shown that early hospital readmissions in decompensated cirrhosis are costly and associated with worse patient outcomes [12].

Therefore, given the growing Hispanic population, and the disparity of the disease burden and the outcomes of HCV within it, we conducted this study in Hispanic patients with $\mathrm{HCV}$ cirrhosis to investigate cirrhosis related complications, to analyze factors associated with readmissions, and to identify any predictors for readmissions.

\section{Patients and methods}

\section{Patient selection and study design}

We retrospectively reviewed the electronic medical records of the hospital admissions of patients with a diagnosis of chronic hepatitis C (ICD-9 codes $070.44,51,54,70$, and 71) at an urban tertiary referral teaching hospital located in the heart of California's San Joaquin Valley between January 1, 2009, and December 31, 2012. Subsequent admissions from the first hospitalization were reviewed and grouped as cirrhosis-related or non-cirrhosis-related. Subgroup analysis was performed on patients with only cirrhosis-related readmissions. Stratification into Hispanic and non-Hispanic ethnicity was based on the demographic data available on chart review. Patients were included in the study if they had lab evidence of hepatitis C by either viral load or antibody to HCV (antiHCV, ORTHO ${ }^{\text {TM }}$ HCV version 3.0 ELISA Test System; Ortho Clinical Diagnostics) or $\mathrm{HCV}$ nucleic acid amplification testing (HCV NAT, Procleix ${ }^{\mathrm{TM}} \mathrm{HIV}-1 / \mathrm{HCV}$ Assay; Gen-Probe Inc., San Diego, CA, USA) and evidence of cirrhosis based on imaging studies, liver biopsy, or clinical documentation of a cirrhosis-related complication. Exclusion criteria were: 1) unavailability of the data pertinent to the study; 2) death or discharge to hospice care; 3 ) liver transplantation prior to or during the discharge hospitalization; 4) other forms of liver disease; and 5) previously treated HCV.

We collected data on the presence or absence of hepatic encephalopathy (HE), upper gastrointestinal bleeding, gastroesophageal varices, ascites, spontaneous bacterial peritonitis (SBP), hepatorenal syndrome (HRS) and hepatocellular carcinoma (HCC) to evaluate the cirrhosisrelated complications in the study groups. Demographic data, available social history, including alcohol use quantified as amount of alcohol used daily, and routine laboratory parameters were reviewed. Child-Turcotte-Pugh (CTP) and model for end-stage liver disease (MELD) scores were calculated. The study was approved by the University of California San Francisco, Fresno Medical Education Program (UCSF Fresno MEP) Institutional Review Board.

\section{Statistical analysis}

Data were tabulated using Microsoft Excel and statistical analysis was performed using Stata, version 11.1. The baseline characteristics of the study populations were compared across the groups using Student's $t$-test for continuous variables and Fischer's exact test or the chi-square test for categorical variables, as needed. The Hispanic subgroup was analyzed for the association of various factors with readmissions. Multivariate logistic regression analysis was performed to identify predictors for readmissions. Statistical significance was set at $\mathrm{P}<0.05$.

\section{Results}

\section{Hispanic vs. non-Hispanic HCV cirrhotic patients}

Of the 813 consecutive patient charts reviewed, 292 met inclusion criteria. Patients were subdivided into Hispanic $(\mathrm{n}=189)$ and non-Hispanic $(\mathrm{n}=103)$ ethnicity groups. Table 1 lists comparisons between these two groups. The groups were largely similar, except that the Hispanic group was younger, with an average age of $53.5 \pm 8.4$ years $(\mathrm{P}=0.03)$, and had a lower albumin level of $3.03 \pm 0.7 \mathrm{~g} / \mathrm{dL}(\mathrm{P}=0.04)$ compared to non-Hispanic patients. Among Hispanics, 55.2\% of females had a body mass index (BMI) $>30 \mathrm{~kg} / \mathrm{m}^{2}$ compared to $35.9 \%$ of males $(\mathrm{P}=0.013)$. Of all Hispanic patients with HRS, $100 \%$ had a $\mathrm{BMI}>30 \mathrm{~kg} / \mathrm{m}^{2}$, compared to $40 \%$ of patients without HRS who had a BMI $>30(\mathrm{P}=0.012)$. Hispanics were also found to have a higher prevalence of decompensated liver disease with variceal hemorrhage compared to nonHispanics $(\mathrm{P}=0.03)$. A history of alcoholism was reported by $67 \%$ of Hispanics compared to $56 \%$ of non-Hispanics $(\mathrm{P}=0.065)$. The distributions of other cirrhosis-related complications (HE, ascites, SBP, HCC, and HRS) were similar between the groups. Various other relevant clinical and laboratory parameters in Hispanic and non-Hispanic patients are listed in Table 1. 
Table 1 Baseline characteristics of the study population (total sample size $=292$ )

\begin{tabular}{|c|c|c|c|}
\hline Characteristics & $\begin{array}{l}\text { Hispanics } \\
(\mathrm{n}=189)\end{array}$ & $\begin{array}{l}\text { Non-Hispanics } \\
\quad(\mathrm{n}=103)\end{array}$ & P-value ${ }^{*}$ \\
\hline Age, mean $\pm S D$ & $53.5 \pm 8.4$ & $55.6 \pm 8.4$ & 0.03 \\
\hline \multicolumn{4}{|l|}{ Sex, n (\%) } \\
\hline Male & $130(68.8)$ & $72(69.9)$ & 0.843 \\
\hline Female & $59(31.2)$ & $31(30.1)$ & \\
\hline $\mathrm{BMI}$, mean $\pm \mathrm{SD}$ & $29.4 \pm 6$ & $28.1 \pm 6.6$ & 0.08 \\
\hline Diabetes, n (\%) & $42(22.2)$ & $34(33)$ & 0.045 \\
\hline Alcohol, n (\%) & $127(67.2)$ & $58(56.3)$ & 0.065 \\
\hline \multicolumn{4}{|l|}{ Labs, mean \pm SD } \\
\hline Albumin & $3.03 \pm 0.7$ & $3.2 \pm 0.7$ & 0.04 \\
\hline AST & $84.3 \pm 67.2$ & $87.8 \pm 111.3$ & 0.74 \\
\hline ALT & $57.4 \pm 42.8$ & $59 \pm 69.3$ & 0.802 \\
\hline Bilirubin & $1.4(0.2-29)$ & $1.1(0.2-15.8)$ & 0.72 \\
\hline Platelets & $91.1 \pm 62.5$ & $103.1 \pm 56.9$ & 0.11 \\
\hline Hemoglobin & $11.5 \pm 5.9$ & $12.1 \pm 7.2$ & 0.51 \\
\hline Creatinine & $1 \pm 0.9$ & $1.21 \pm 1$ & 0.06 \\
\hline $\mathrm{HbAlC}$ & $6.01 \pm 1.9$ & $6.1 \pm 1.4$ & 0.79 \\
\hline INR & $1.52 \pm 0.7$ & $1.4 \pm 0.6$ & 0.12 \\
\hline MELD, mean \pm SD & $12.2 \pm 6.1$ & $12 \pm 6.8$ & 0.84 \\
\hline $\mathrm{CTP}$, mean $\pm \mathrm{SD}$ & $8.3 \pm 2.5$ & $7.9 \pm 2.2$ & 0.18 \\
\hline CTP class A & $55(29.1)$ & $31(30.1)$ & 0.33 \\
\hline CTP class B & $83(43.9)$ & $52(50.5)$ & \\
\hline CTP class $\mathrm{C}$ & $51(27)$ & $20(19.4)$ & \\
\hline No. of complications $(>1)$ & $54(52.4)$ & $112(59.3)$ & 0.447 \\
\hline \multicolumn{4}{|l|}{ History of complications } \\
\hline HE & $65(34.4)$ & $32(31.1)$ & 0.57 \\
\hline Ascites & $117(61.9)$ & $66(64.1)$ & 0.71 \\
\hline SBP & $24(12.7)$ & $7(6.8)$ & 0.12 \\
\hline Variceal hemorrhage & $125(66.1)$ & $55(53.4)$ & 0.03 \\
\hline $\mathrm{HCC}$ & $17(9)$ & $10(9.71)$ & 0.84 \\
\hline HRS & $5(2.7)$ & $6(5.8)$ & 0.15 \\
\hline \multicolumn{4}{|l|}{ Insurance } \\
\hline Medicare/private & $46(24.3)$ & $34(33)$ & 0.112 \\
\hline Medical/MISP/No ins & $143(75.7)$ & $69(67)$ & \\
\hline Previous EGD & $83(43.9)$ & $36(35)$ & 0.14 \\
\hline Prophylactic BB & $61(32.3)$ & $37(35.9)$ & 0.31 \\
\hline
\end{tabular}

${ }^{*} \mathrm{P}$ values calculated using Student's $t$-test for continuous variables and Fischer's exact/Chi-square test for categorical variables.

BMI, body mass index; AST, aspartate aminotransferase; ALT, alanine aminotransferase; $H b A 1 C$, glycosylated hemoglobin; INR, international normalized ratio; $M E L D$, model for end-stage liver disease; CTP,

Child-Turcotte-Pugh; HE, hepatic encephalopathy; SBP, spontaneous bacterial peritonitis; HCC, hepatocellular carcinoma; HRS, hepatorenal syndrome; $E G D$, esophagogastroduodenoscopy; $B B, \beta$-blocker

\section{Readmissions in Hispanic HCV cirrhosis study population}

In total, there were 136 cirrhosis-related readmissions, of which the majority (71\%) were Hispanics, while only $29 \%$ were non-Hispanics $(\mathrm{P}=0.035)$. Approximately $70 \%$ of the Hispanics were in CTP class B or C. Among Hispanics, HE and esophageal variceal hemorrhage were the most frequent causes for the first and subsequent readmissions. Hispanics with readmissions had higher CTP class (B and C) (Fig. 1) and MELD scores $(\geq 15)$ (Fig. 2), and a higher rate of alcohol use, HE, spontaneous bacterial peritonitis, HCC, and variceal hemorrhage $(\mathrm{P}<0.05$, Table 2). The presence of two or more cirrhosis-related complications was significantly associated with readmission.

Logistic regression analysis was carried out to identify the predictors for cirrhosis-related readmissions. With readmissions as the dependent variable, predictors for the multivariate model were identified based on known clinical predictions and those with a P-value $<0.25$ on univariate analysis. Multivariate logistic regression analysis identified alcohol use (odds ratio [OR] 2.63; 95\% confidence interval [CI] 1.1-6.4], HE (OR 5.5; 95\%CI 2-15.3), variceal hemorrhage (OR 3.2; 95\%CI 1.3-8.2), and CTP class (OR 3.3; 95\%CI 1.4-8.1) as predictors for readmission among Hispanics in our study sample (Table 3, Fig. 3). There

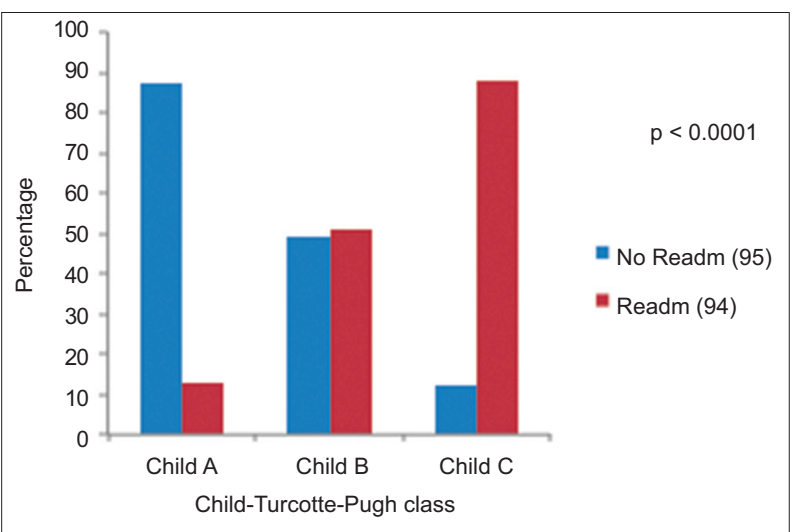

Figure 1 Child-Turcotte-Pugh class distribution among Hispanics with hepatitis $\mathrm{C}$ virus cirrhosis-related admissions

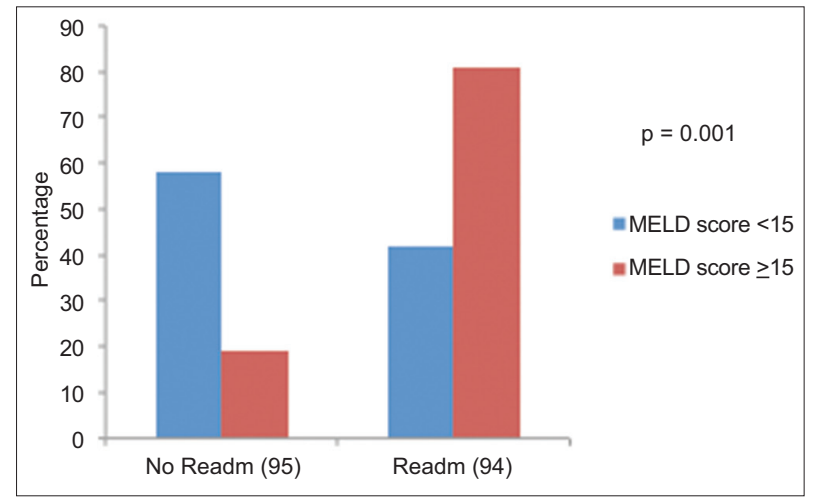

Figure 2 Model for end-stage liver disease score distribution among Hispanics with hepatitis $C$ virus-related cirrhosis 
was no statistically significant difference between Hispanics with or without readmissions with regard to type of health insurance, prophylactic $\beta$-blocker use, or diabetes.

\section{Discussion}

Hispanics have a disproportionately high prevalence of $\mathrm{HCV}$ infection compared to other ethnic groups [3,5,7]. For

Table 2 Association of various factors with gastrointestinal-related readmissions among Hispanics

\begin{tabular}{cccc}
\hline Variable & $\begin{array}{c}\text { Readmissions } \\
(94)\end{array}$ & $\begin{array}{c}\text { No } \\
\text { readmissions }\end{array}$ & P-value \\
& &
\end{tabular}

(95)

\begin{tabular}{|c|c|c|c|}
\hline Age, years & $53.4 \pm 8.7$ & $53.7 \pm 8$ & 0.798 \\
\hline Male & $68(52.3)$ & $62(47.8)$ & 0.347 \\
\hline Female & $26(44.1)$ & $33(55.9)$ & \\
\hline BMI, kg/m² & $29.1 \pm 6.3$ & $29.8 \pm 5.7$ & 0.415 \\
\hline $\begin{array}{l}\text { MELD score } \\
<15 \\
\geq 15\end{array}$ & $\begin{array}{l}13.1 \pm 6.7 \\
65(42.3) \\
29(80.6)\end{array}$ & $\begin{array}{c}9.8 \pm 3.2 \\
88(57.5) \\
7(19.4)\end{array}$ & $\begin{array}{c}0.001 \\
<0.0001\end{array}$ \\
\hline $\begin{array}{c}\text { CTP score } \\
\text { Class A } \\
\text { Class B } \\
\text { Class C }\end{array}$ & $\begin{array}{c}9.7 \pm 2.4 \\
7(12.7) \\
42(50.6) \\
45(88.2)\end{array}$ & $\begin{array}{c}6.9 \pm 1.7 \\
48(87.3) \\
41(49.4) \\
6(11.8)\end{array}$ & $<0.0001$ \\
\hline $\begin{array}{l}\text { History of chronic } \\
\text { alcohol use }\end{array}$ & $72(56.7)$ & $55(43.3)$ & 0.008 \\
\hline History of DM & $19(45.2)$ & $23(54.8)$ & 0.600 \\
\hline History of HE & $56(86.2)$ & $9(13.8)$ & $<0.0001$ \\
\hline History of ascites & $75(64.1)$ & $42(35.9)$ & $<0.0001$ \\
\hline $\begin{array}{l}\text { History of varices } \\
\text { hemorrhage }\end{array}$ & $69(55.2)$ & $44.8(44.8)$ & 0.046 \\
\hline History of SBP & $23(95.8)$ & $1(4.2)$ & $<0.0001$ \\
\hline History of HCC & $14(82.4)$ & $3(17.7)$ & 0.023 \\
\hline History of HRS & $4(80)$ & $1(20)$ & 0.568 \\
\hline Medicare/private & $21(45.6)$ & $25(54.4)$ & 0.612 \\
\hline Med-cal/none & $73(51.1)$ & $40(48.9)$ & \\
\hline $\begin{array}{l}\text { Complications } \\
\text { None } \\
\text { One } \\
\text { Two }\end{array}$ & $\begin{array}{c}0(0) \\
12(23.1) \\
81(72.3)\end{array}$ & $\begin{array}{c}24(100) \\
40(76.9) \\
31(31)\end{array}$ & $<0.0001$ \\
\hline Previous EGD & $47(56.6)$ & $36(43.4))$ & 0.108 \\
\hline Prophylactic BB & $34(55.7)$ & $27(44.3)$ & 0.163 \\
\hline Prophylactic antibiotics & $4(80)$ & $1(20)$ & 0.181 \\
\hline
\end{tabular}

${ }^{*} \mathrm{P}$ values calculated using Student's $t$-test for continuous variables and Fischer's exact/Chi-square test for categorical variables.

$B M I$, body mass index; AST, aspartate aminotransferase; ALT, alanine aminotransferase; HbA1C, glycosylated hemoglobin; INR, international normalized ratio; $M E L D$, model for end-stage liver disease; CTP, Child-Turcotte-Pugh; HE, hepatic encephalopathy; SBP, spontaneous bacterial peritonitis; HCC, hepatocellular carcinoma; HRS, hepatorenal syndrome; $E G D$, esophagogastroduodenoscopy; $B B, \beta$-blocker patients with chronic liver disease, those with HCV-related admissions have been shown to have the most pronounced increase in cost [15]. The rise in overall healthcare costs has led to an increased focus on readmission rates and the need to identify related issues to improve quality of care and costeffectiveness. This is the first study we know of to investigate $\mathrm{HCV}$ cirrhosis-related readmissions in Hispanics and identify predictors for readmission.

Earlier studies have shown that re-hospitalizations among patients with cirrhosis are common and, are related to liver disease itself and portal hypertension. However, these studies have had predominately non-Hispanic populations $[12,16]$. In 2003, Nguyen et al reported a growing burden of hepatitis C-related liver disease in a study using a Nationwide Inpatient Sample (NIS). They reported that the inflation-adjusted total economic burden of decompensated cirrhosis increased from $\$ 1.15$ billion to $\$ 2.1$ billion over a 6 -year period $(\mathrm{P}=0.003)$ [15].

A study by Volk et al that included $82 \%$ Caucasian patients with HCV cirrhosis identified MELD score, serum sodium, and number of medications [12] as risk factors for readmissions. In a study by Berman et al, with a population that was $83 \%$ Caucasian and included all causes of liver cirrhosis, the predictors for readmission were found to be male sex, the presence of diabetes, and MELD score [17]. Studies in minorities, such as one by Ganesh et al, showed that African Americans with cirrhosis had a higher number of hospital readmissions, though the study was not designed to identify predictors [16]. Our study addressed these shortcomings, as it included predominately Hispanics and identified variceal hemorrhage, HE, and CPT class as predictors for hospital readmissions.

Prior studies have shown variceal hemorrhage to be an important predictor for re-admissions, and Hispanics have a higher incidence of de novo varices [9]. While the presence of varices can indicate a more aggressive course and longer disease duration, it also provides important prognostic information. Studies in patients with compensated HCV-related liver disease have shown that the presence of varices at baseline was associated with a higher rate of mortality, HCC, and

Table 3 Multivariate analysis of predictors for cirrhosis related readmissions among Hispanic patients with hepatitis $\mathrm{C}$ virus-related cirrhosis

\begin{tabular}{lll}
\hline Predictor variable & Odds ratio (95\%CI) & P-value \\
\hline Alcohol use & $2.63(1.1-6.4)$ & 0.034 \\
Hepatic encephalopathy & $5.5(1.97-15.3)$ & 0.001 \\
Varices & $3.2(1.25-8.2)$ & 0.015 \\
Ascites & $1.5(0.57-3.9)$ & 0.417 \\
SBP & $9.4(0.9-98.5)$ & 0.062 \\
HCC & $4.7(0.95-23.3)$ & 0.058 \\
CTP class & $3.3(1.4-8.1)$ & 0.009 \\
MELD score & $1.03(0.9-1.14)$ & 0.542 \\
\hline CTP, Child-Turcotte-Pugh; MELD, model for end-stage liver disease; SBP, \\
spontaneous bacterial peritonitis; HCC, hepatocellular carcinoma
\end{tabular}




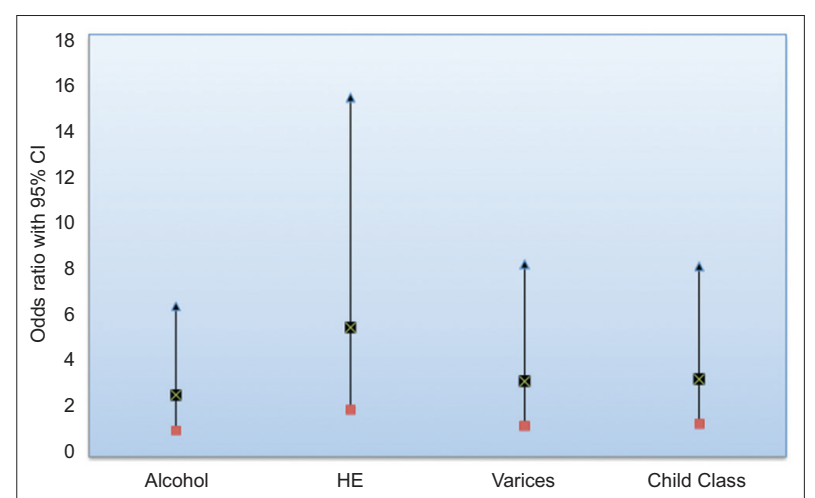

Figure 3 Predictors for readmissions among Hispanics with hepatitis $\mathrm{C}$ virus cirrhosis

subsequent decompensation compared to patients who had a similar class of disease without varices $[10,11,18]$. While these studies underscore the importance of variceal screening and prophylactic treatment in HCV cirrhosis, Hispanic ethnicity in such patients amounts to a double hit, especially with respect to poor outcomes. The overall picture may well be further confounded by the socioeconomic burden in this group. In addition, the study sample comprised about $35 \%$ patients with CTP class C in the readmissions group compared to only $6 \%$ in no-readmissions group (Fig. 1); thus, the higher CTP class could be contributing to readmissions.

$\mathrm{HE}$ is a common cause for initial hospitalization in patients with cirrhosis and, the hospitalization rates from $\mathrm{HE}$ are increasing $[12,15,16]$. In a study by Ganesh et al, HE was noted to be one of the most common causes for initial admission in patients with cirrhosis who had multiple admissions [16]. Another study found $\mathrm{HE}$ to be one of the most frequent reasons for potentially preventable readmissions [12]. HE is associated not only with decompensation and hospitalization, but also with poor prognosis and survival [19]. In our study, we found $\mathrm{HE}$ to be one of the most common reasons for first and subsequent readmissions and we confirmed its prognostic significance. CTP scores were also a statistically significant predictor of readmission for Hispanics, as HE is a component in the calculation of CTP scores. In addition, MELD scores were significantly higher in the readmissions group, as seen in previous studies $[12,16]$, but were not predictors of readmission on multivariate regression analysis.

In addition to variceal hemorrhage and HE, our study found that readmissions were significantly associated with alcoholism, HCC, SBP, and two or more cirrhosis-related complications. No difference in the prevalence of ascites or the numbers of those taking prophylactic $\beta$-blockers was noted in the study population (Table 2). We also found that all of the Hispanic patients diagnosed with HRS were obese, with a BMI over 30; this could have been due to coexistent nonalcoholic steatohepatitis in these patients, as they tend to have more renal dysfunction [20].

This was a single-center retrospective study with inherent limitations and biases. The etiology of HCV cirrhosis-related readmissions, as defined earlier, may have underestimated the total readmissions, as other causes, such as volume overload,

\section{Summary Box}

\section{What is already known:}

- Hospital readmissions in patients with cirrhosis are a major healthcare burden

- Readmissions in decompensated cirrhosis are associated with worse patient outcomes

- Hispanics have worse hepatitis C virus (HCV)related disease outcomes with higher rates of progression

\section{What the new findings are:}

- Advanced Child-Turcotte-Pugh class is a predictor for readmissions among Hispanics with HCVrelated cirrhosis

- Variceal hemorrhage and hepatic encephalopathy contribute to the majority of readmissions

- Better disease management interventions with a focus on prevention are needed

infections, etc., could also be cirrhosis-related. The higher rates of alcoholism in Hispanics could also be a contributing factor to readmissions. Because of the retrospective nature of the study, social factors influencing readmissions, such as financial status, social support, etc., were not explored and will need to be addressed in future prospective studies. Additionally, the study cohort included patients who had documented evidence of HCV infection, whereas there may be other factors that could have accelerated progression to cirrhosis in this population, in particular alcohol use and metabolic syndrome.

In conclusion, CTP classes B and C, among other factors, were the major predictors for readmissions in Hispanics with $\mathrm{HCV}$-related cirrhosis. The fact that the majority of these readmissions were due to $\mathrm{HE}$ and variceal hemorrhage warrants a shift in focus towards preventing these complications in this select minority group. Better disease management interventions with a focus on prevention, especially with regard to alcohol use, and the allocation of resources to improve cost-effectiveness and quality of care are the need of the hour.

\section{References}

1. Day JC. Population projections of the United States by age, sex, race, and Hispanic origin: 1995 to 2050. U.S. Bureau of the Census, Current Population Reports 1996;25-1130.

2. Heron M. Deaths: leading causes for 2007. Natl Vital Stat Rep 2011;59:1-95.

3. Vong S, Bell BP. Chronic liver disease mortality in the United States, 1990-1998. Hepatology 2004;39:476-483.

4. Kim WR. The burden of hepatitis C in the United States. Hepatology 2002;36:S30-S34. 
5. Wise M, Bialek S, Finelli L, Bell BP, Sorvillo F. Changing trends in hepatitis C-related mortality in the United States, 1995-2004. Hepatology 2008;47:1128-1135.

6. Davis GL, Albright JE, Cook SF, Rosenberg DM. Projecting future complications of chronic hepatitis C in the United States. Liver Transpl 2003;9:331-338.

7. Alter MJ, Kruszon-Moran D, Nainan OV, et al. The prevalence of hepatitis C virus infection in the United States, 1988 through 1994. N Engl J Med 1999;341:556-562.

8. Verma S, Bonacini M, Govindarajan S, Kanel G, Lindsay KL, Redeker A. More advanced hepatic fibrosis in hispanics with chronic hepatitis C infection: role of patient demographics, hepatic necroinflammation, and steatosis. Am J Gastroenterol 2006;101:1817-1823.

9. Fontana RJ, Sanyal AJ, Ghany MG, et al. Factors that determine the development and progression of gastroesophageal varices in patients with chronic hepatitis C. Gastroenterology 2010;138:23212331, 2331.e1-e2.

10. Bruno S, Zuin M, Crosignani A, et al. Predicting mortality risk in patients with compensated HCV-induced cirrhosis: a long-term prospective study. Am J Gastroenterol 2009;104:1147-1158.

11. Gomez EV, Rodriguez YS, Bertot LC, et al. The natural history of compensated HCV-related cirrhosis: a prospective long-term study. J Hepatol 2013;58:434-444.

12. Volk ML, Piette JD, Singal AS, Lok AS. Chronic disease management for patients with cirrhosis. Gastroenterology 2010;139:14-16.e1.
13. Talwalkar JA. Prophylaxis with beta blockers as a performance measure of quality health care in cirrhosis. Gastroenterology 2006;130:1005-1007.

14. Jencks SF, Williams MV, Coleman EA. Rehospitalizations among patients in the Medicare fee-for-service program. $N$ Engl J Med 2009;360:1418-1428.

15. Nguyen GC, Segev DL, Thuluvath PJ. Nationwide increase in hospitalizations and hepatitis $\mathrm{C}$ among inpatients with cirrhosis and sequelae of portal hypertension. Clin Gastroenterol Hepatol 2007;5:1092-1099.

16. Ganesh S, Rogal SS, Yadav D, Humar A, Behari J. Risk factors for frequent readmissions and barriers to transplantation in patients with cirrhosis. PLoS One 2013;8:e55140.

17. Berman K, Tandra S, Forssell K, et al. Incidence and predictors of 30-day readmission among patients hospitalized for advanced liver disease. Clin Gastroenterol Hepatol 2011;9:254-259.

18. 'D’Amico G, Garcia-Tsao G, Pagliaro L. Natural history and prognostic indicators of survival in cirrhosis: a systematic review of 118 studies. J Hepatol 2006;44:217-231.

19. Bustamante J, Rimola A, Ventura PJ, et al. Prognostic significance of hepatic encephalopathy in patients with cirrhosis. J Hepatol 1999;30:890-895.

20. Park CW, Tsai NT, Wong LL. Implications of worse renal dysfunction and medical comorbidities in patients with NASH undergoing liver transplant evaluation: impact on MELD and more. Clin Transplant 2011;25:E606-E611. 